\title{
Comparison of the Online Education Platforms, and Innovative Solution Proposals
}

\author{
Yilmaz S. ${ }^{2}$ \\ Erol İ.E. ${ }^{1}$
}

\author{
${ }^{2}$ Serhat YILMAZ, İstanbul Aydın University, (Turkey), \\ e-mail: serhatyilmaz@aydin.edu.tr \\ ${ }^{1}$ Ihsan Emre EROL, İstanbul Aydın University, (Turkey). \\ e-mail: iemreerol@aydin.edu.tr
}

\begin{abstract}
The traditional education methods may remain incapable to meet the today's rising education demands by reason of the fact that it depends on a time, a place, and a person. On the other hand, the training programs in the online learning environments offer important advantages for meeting the rising education demands, and have been become more popular. However, the online education programs that have become more and more popular have disadvantages such as the limitation of instructor control, the boringness due to monotony and the lack of experiential learning. These disadvantages caused by the lack of platforms where online education programs are available, are being minimized by the help technological developments. In this regard, it has been needed to research what kind of disadvantages may arise based on the lack of platforms, and to create innovative solutions in the light of developing technology against these disadvantages.

In line with this requirement, under the scope of this study, a comparative content analyze is performed on the most popular platforms (synchronous and asynchronous and blended) that are preferred by universities in Turkey, and it has also been examined that how these online educations can be transformed into a more effective structure with the help of developing technologies. As a result of this study it has been determined that innovative technologies such as Augmented Reality and Virtual Reality make significant contributions to the instructor control, interaction and the experiential learning (transforming theory into practice) in online educations.
\end{abstract}

Keywords: Online Education, e-Learning, Webinar, e-Learning Platforms.

\section{Introduction}

All research of human history showing us; humans are living small groups for protecting them self to external threats. Another human kind attribute is educational activities for educational requirement to young people as a requirement of collective life. In old communities these educational activities are on the just fundamentals of life. But in the historical development; education activities changed to interdisciplinary structure and divided into several subjects.

The historical evolution of educational activities continues with communication technologies. In this evolution process; most visible attribute is providing of individuals can take any education at any place by remote access to education institutions. As a though; the first distance education examples were found in the 1700 s with letters, magazines and books. But multimedia based and interactive distance learning Works coincide of early days of 21 st century. Distance learning Works of 21 st century is opportunity for growing human population educational demands. With this development and success of distance education, caused at increasing distance education and traditional education comparison discussions. But despite long time discussions; in the close history, distance education works are spread rapidly. For example; in the USA only in 2011, more than 100 distance education institutions are accredited and 2 million individuals receive education. Other way; more than 40 distance undergraduate level education programs are approved (Engin, 2013). 
Today; because of opportunities of the distance educations and rapid developments in communication technologies and especially the use of online technologies like internet for the distance learning; many respected educational institutions are started own distance learning systems and off course this situation has led to the emergence of a competitive environment. In this competitive environment; naturally, many of online distance education platforms developed. With this reality; how to serve online distance education programs more effective and witch platforms more useful for this objective question are born. As a result of all these; on the one hand continuation of discussions and the other hand rapid spread of the educational activities with this style; revealed the need for research in this field.

In this context; comparing distance online education platforms and revealing their disadvantages; provides important contributions to the positive impact of the educations provided by this method and distance online education field. With this research; most preferred distance online education platforms content analysis of universities of Turkey and answering how to turn more effective structure with today's emerging technologies question.

\section{Literature}

In early days of history; humans are created natural information stream with between individuals due to human's community life requirement. Believed to be as old as humanity education activities, systematically stream process of this information. We don't know what is the time of starting these activities. But after the invention of writing, all this information accessible (Arslan, 2009: 28). The invention of the writing is a most important milestone for education activities off course below other effects of culture, geography and prosperity. The simplest form; defined as process of behavior change education (Şişman, 2007:7) has evolved, changed and improved itself over the ages (Arslan, 2009: 27). Last one of the education activities historical development, connected with development of communication technologies in the 21st century; development of communication technologies that causes radical changes in every part of life, providing to us communications with audio and video (İşman, 2011: 2). But; distance learning activities discussions based on improve or worse learning have been going on for a long time (Beynon, 2007; Clark, 2001). From the first years of distance education to the present day, based on communication technologies with these discussions, distance education effectiveness has been questioned (Şimşek, 2012).

As a concept, research of distance education some sources based on 1700s (İşman, 2011: 14) other sources based on 1800st (Saba, 2003: 3); can be summarized shortly. From a wide angle of distance education; researcher of working this field adopt two different approach (Schlosser, Anderson, 1994: 7). B"orje Holmberg, Charles A. Wedemeyer and Michael G. Moore are known as pioneering theorists of conceptual approach. In this approach, student and student interactive education process are center and this situation is distinguishing feature of distance educations. On the other hand; Desmong Keegan, Otto Peters, Randy Garrison and John Anderson are known as pioneering theorists of structural approach. With this approach focusing, industrialism issues and effect on education process of these issues without losing student centrality (Saba, 2003: 4). From historical development of distance learning, then four different classification revealed. In this classification, first stage is learning model using letters. Second stage is called multi-media model. In this model, pressed materials and electronic communication devices like radio and television used for education. Third stage a synchronous model called by tel-learning. Fourth stage of distance learning is flexible learning model. With this model, using of internet comes to the fore (Aşkar, 2003).

Fourth stage of distance education that became spread in the late of 20th century, observed essential changes of distance learning tools, distribution and methods with spread of computers and internet technologies (Epignosis LLC, 2014: 8). In this development process; individuals gained access for many information and learning opportunities with virtual learning environments (Preece, vd 2003). Final of these development; at the present day, online education activities giving communication capability for learner and teachers. A student in a country of the world can learn from another teacher of another country of the world via audio, video communication network and some universities giving educations to students from different countries of the world with called by "open 
university" structures. Today; increasing education demands, diversification of educational needs, individualization of education and become many educational applications feasible through distance education comes to fore as determining factors off course in the light of technological development. (Işman, 2011: 4) There are some issues to be considered for complex teaching and learning processes through online systems because of includes individuals of different skills, expectations and demands (Campanella, et al., 2008). Each of these issues should be considered as a seperate research topic. But in this research; based on surveying technological issues because of comparing online education platforms.

Learning Management Systems and E-Learning Author Tools are technological issues to be considered. (Aşkar, 2003; Watson \& Watson, 2007). Learning Management Systems (LMS) are computer softwares for publishing content at the same time education recording and managing, skill tracking, analysing and reporting (Watson \& Watson, 2007). Other hand, E- Learning Author Tools are advanced softwares for creating e learning content like contentinteractive presentations, educational presentations. (Özkeskin, 2007)

LMS and e-learning author tools software's must be including some parameters for technological issues. We can classify these parameters as follows (Campanella, et al., 2008; Aslan, 2013; Bayram, vd. 2009; Growth Engineering; E-Learning Industry; Trivantis; Uzaktan Eğitim, Öğrenme Yönetim Sistemi (LMS); Edutechnica); System Parameters: Authentication and security, gamification, support of power point, availability of storyboard, conference manager, content library, content manager, data import and export, data manager, document manager, installation of system (hosted, cloud or local installation), licensing (free, trial, paid), maintenance and backup, availability of API (Application Programming Interface), mobile application, user interface with multiple language support, education system with multiple language support, live streaming capabilities, multimedia environment, multiple export formats, platform independence, notifications with e-mail, notifications with sms, podcast manager, education content manager system - LCMS, interactive lessons. Interface Parameters: Mobile friendly interface, customizable interface, user friendly interface. Educational and Administrative: Reporting tools, rating tools, certificate manager, virtual class manager, cooperation manager, lecturer's planner, user access controllers, course system, simulations, curriculum management, lesson ratings, availability of the glossary of terms, development monitoring, activity manager, quiz manager, education criterion and outputs, wish lists, support of webinar. Harmony Parameters: Third party authoring tools, third party teleconference tools, availability of Tin Can Api, AICC, SCORM. Customizable Parameters: Customizable fields, customizable functions, customizable reporting's. Student Parameters: Lesson register system, selfcontrolled education, social learning, student manager, student register system, student portal. ELearning Types: Offline learning, online learning. Learning Models: Synchronous learning, asynchronous learning, blended learning.

\section{Research}

Flexible learning model, the fourth stage of distance education and starting with the use of the internet allows giving more effective distance learning and rapid spreading together with development of communication technologies. With these improvements, distance education can be online and used as a preferred method by reputable educational institutions. This situation increasing platforms of online education systems and creating competition environment between platforms and education institutions. At the final, created research fields for how to transferring information more effective with online education programs and identification of advantages and disadvantages provide by or produce educational content platforms. In this context; disadvantages of the lack of online education platforms and what need to produce innovative solutions in the light of developing technologies revealed. With this research to this requirement, performed comparative analysis of online education platforms and researching these online educations how to turn more effective structure with emerging technologies. The scope of this research, universities of Turkey's most preferred LMS platforms Moodle, Canvas, Blackboard and Sakai determined in order. At the research; developing their own platforms or unknown LMS platforms were excluded from the scope because of content analysis is not possible. In the scope of the research, surveyed LMS platforms can obtain e-Learning Author tools contents and produce own contents. In the content analysis Articulate Storyline because of global market share and Adobe Captivate E-Learning Author Tool due to support of virtual reality has been examined with all 
other criteria (Market Insight Reports). The following hypotheses were developed within the scope of the research;

Hypotheses 1: Platforms of using online education programs have similar features and capabilities.

Hypotheses 2: Platforms of using online education programs have innovative media technologies like virtual reality, augmented reality, mixed reality.

Hypotheses 3: Platforms of using online education programs have limitations of live streaming like webinar.

Hypotheses 4: Platforms of using online education programs have limitations of mobile applications.

Hypotheses 5: Authoring tools softwares for produce online education programs have not innovative media technologies like virtual reality, augmented reality, mixed reality.

Hypotheses 6: Authoring tools softwares for produce online education programs have similar features and capabilities.

Hypotheses 7: Authoring tools softwares for produce online education programs have not compatibility issues.

Hypotheses 8: Authoring tools softwares for produce online education programs and platforms of using online education programs; compatible with online educatiın standarts like SCORM and AICC.

\begin{tabular}{|c|c|c|c|c|c|c|c|}
\hline & & Learni & ig Manage & ment Sy & stems & $\begin{array}{r}\text { E - Learn } \\
\text { To }\end{array}$ & $\begin{array}{l}\text { ing Author } \\
\text { ols }\end{array}$ \\
\hline & & $\begin{array}{l}\frac{0}{\overline{0}} \\
\frac{0}{\Sigma}\end{array}$ & 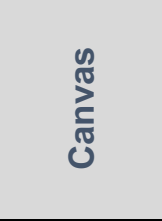 & 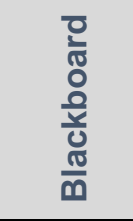 & " & 造 & 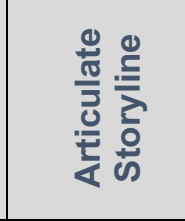 \\
\hline & $\begin{array}{l}\text { Authentication and } \\
\text { Security }\end{array}$ & $\checkmark$ & $\checkmark$ & $\checkmark$ & $\checkmark$ & $\checkmark$ & $\checkmark$ \\
\hline & Gamification & $\checkmark$ & $\checkmark$ & $\sqrt{ }$ & $\mathrm{x}$ & $\checkmark$ & $\checkmark$ \\
\hline & Support of Powerpoint & $\checkmark$ & $\checkmark$ & $\checkmark$ & $x$ & $\checkmark$ & $\checkmark$ \\
\hline & Availability of Storyboard & $x$ & $x$ & $x$ & $x$ & $\checkmark$ & $\checkmark$ \\
\hline & Conference Manager & $x$ & $\checkmark$ & $\checkmark$ & $x$ & $\checkmark$ & $x$ \\
\hline System & Content Library & $x$ & $x$ & $\checkmark$ & $x$ & $\checkmark$ & $\checkmark$ \\
\hline Parameters & Content Manager & $\checkmark$ & $\checkmark$ & $\checkmark$ & $\checkmark$ & $\checkmark$ & $\checkmark$ \\
\hline & Data Import and Export & $\checkmark$ & $\checkmark$ & $\checkmark$ & $\checkmark$ & $\checkmark$ & $\checkmark$ \\
\hline & Data Manager & $\checkmark$ & $\checkmark$ & $\checkmark$ & $\checkmark$ & $\checkmark$ & $\checkmark$ \\
\hline & Document Manager & $\checkmark$ & $\checkmark$ & $\checkmark$ & $\checkmark$ & $\checkmark$ & $\checkmark$ \\
\hline & $\begin{array}{l}\text { Installation of } \\
\text { System(Hosted, cloud, } \\
\text { local installation) }\end{array}$ & $\begin{array}{l}\text { Hosted, } \\
\text { Cloud }\end{array}$ & $\begin{array}{l}\text { Hosted, } \\
\text { Cloud }\end{array}$ & $\begin{array}{l}\text { Hosted, } \\
\text { Cloud }\end{array}$ & $\begin{array}{l}\text { Hosted, } \\
\text { Cloud }\end{array}$ & $\begin{array}{c}\text { Local } \\
\text { installation }\end{array}$ & $\begin{array}{c}\text { Local } \\
\text { installation }\end{array}$ \\
\hline & $\begin{array}{l}\text { Licensing (free, trial, open } \\
\text { source, paid) }\end{array}$ & Paid & $\begin{array}{l}\text { free up to } \\
\text { a certain } \\
\text { number } \\
\text { of users }\end{array}$ & Paid & Free & $\begin{array}{l}\text { Paid/ } \\
\text { Trial }\end{array}$ & $\begin{array}{c}\text { Paid/ } \\
\text { Trial }\end{array}$ \\
\hline & Maintenance \& Backup & Manual & Manual & Manual & Manuel & $\begin{array}{l}\text { Manual/ } \\
\text { Automatic }\end{array}$ & $\begin{array}{l}\text { Manual/ } \\
\text { Automatic }\end{array}$ \\
\hline & Availability of API & $\checkmark$ & $\checkmark$ & $\checkmark$ & $\checkmark$ & $\checkmark$ & $\checkmark$ \\
\hline
\end{tabular}




\begin{tabular}{|c|c|c|c|c|c|c|}
\hline Mobile Application & $\checkmark$ & $x$ & $\checkmark$ & $x$ & $x$ & $x$ \\
\hline $\begin{array}{l}\text { User Interface With } \\
\text { Multiple Language Support }\end{array}$ & $\checkmark$ & $\checkmark$ & $\checkmark$ & $\checkmark$ & $\checkmark$ & $\sqrt{ }$ \\
\hline $\begin{array}{l}\text { Education System With } \\
\text { Multiple Language Spport }\end{array}$ & $\sqrt{ }$ & $x$ & $\sqrt{ }$ & $x$ & $\sqrt{ }$ & $\sqrt{ }$ \\
\hline $\begin{array}{l}\text { Live Streaming } \\
\text { Capabilities }\end{array}$ & $x$ & $x$ & $\checkmark$ & $x$ & $x$ & $x$ \\
\hline Multimedia Environment & $\checkmark$ & $\checkmark$ & $\checkmark$ & $\checkmark$ & $\checkmark$ & $\sqrt{ }$ \\
\hline Multiple Export Formats & $x$ & $x$ & $x$ & $x$ & $\checkmark$ & $\sqrt{ }$ \\
\hline Platform Independence & $\checkmark$ & $\sqrt{ }$ & $\sqrt{ }$ & $\checkmark$ & $x$ & $\mathrm{x}$ \\
\hline Notifications - Email & $\sqrt{ }$ & $\sqrt{ }$ & $\sqrt{ }$ & $\checkmark$ & $\sqrt{ }$ & $\sqrt{ }$ \\
\hline Notifications - SMS & $x$ & $x$ & $\checkmark$ & $x$ & $x$ & $x$ \\
\hline Podcast Manager & $x$ & $\checkmark$ & $x$ & $\checkmark$ & $\mathrm{x}$ & $\checkmark$ \\
\hline $\begin{array}{l}\text { Education Content } \\
\text { Manager System - LCMS }\end{array}$ & $\checkmark$ & $\checkmark$ & $\checkmark$ & $\checkmark$ & $\checkmark$ & $\checkmark$ \\
\hline Interactive Lessons & $x$ & $x$ & $x$ & $\checkmark$ & $\checkmark$ & $\sqrt{ }$ \\
\hline
\end{tabular}

Table 1: LMS and E - Learning Author Tools System Parameters Comparison

Content analysis conducted within the scope of system parameters; LMS platforms of Moodle, Canvas, Blackboard and Sakai has the parameters of authentication and security, content manager, data import and export, data manager, document manager, installation of system, maintenance and backup, availability of api, user interface with language support, multimedia environment, platform independence, notifications -email and education content manager system - Icms. Adobe Captivate and Articulate Storyline has differences with podcast manager and conference manager other parameters showing us these platforms are carrying similar features.

\begin{tabular}{|c|c|c|c|c|c|c|c|}
\hline & & \multicolumn{4}{|c|}{ Learning Management Systems } & \multicolumn{2}{|c|}{$\begin{array}{l}\text { E - Learning } \\
\text { Author Tools }\end{array}$} \\
\hline & & 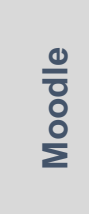 & 疍 & $\begin{array}{l}\text { 음 } \\
\frac{0}{0} \\
\frac{0}{0} \\
\frac{0}{0} \\
\frac{\pi}{0}\end{array}$ & 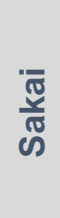 & 융 & 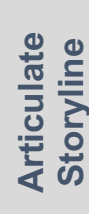 \\
\hline \multirow{3}{*}{$\begin{array}{l}\text { Interface } \\
\text { Parameters }\end{array}$} & Mobile Friendly Interface & $\checkmark$ & $\checkmark$ & $\checkmark$ & $\checkmark$ & $\checkmark$ & $\checkmark$ \\
\hline & Customizable Interface & $\checkmark$ & $\checkmark$ & $\mathrm{x}$ & $\mathrm{x}$ & $\checkmark$ & $\checkmark$ \\
\hline & User Friendly Interface & $\mathrm{x}$ & $\checkmark$ & $\mathrm{x}$ & $\mathrm{x}$ & $\mathrm{x}$ & $\checkmark$ \\
\hline
\end{tabular}

Table 2: LMS and E - Learning Author Tools Interface Parameters Comparison

For 4 LMS platforms have mobile friendly interface. Adobe Captivate and Articulate Storyline comparisons are revealed they haven't got user friendly interface. LMS and Authoring Tools comparisons are showing us only mobile friendly interface is common feature. 


\begin{tabular}{|c|c|c|c|c|c|c|c|}
\hline & & \multicolumn{4}{|c|}{ Learning Management Systems } & \multicolumn{2}{|c|}{$\begin{array}{l}\text { E - Learning } \\
\text { Author Tools }\end{array}$} \\
\hline & & $\begin{array}{l}\stackrel{0}{\bar{\Xi}} \\
\stackrel{\circ}{\Sigma}\end{array}$ & 疍 & $\begin{array}{l}\text { 일 } \\
\frac{0}{0} \\
\frac{0}{0} \\
\frac{0}{0} \\
\frac{\pi}{n}\end{array}$ & 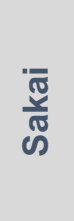 & 造 & 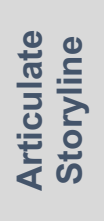 \\
\hline \multirow{18}{*}{$\begin{array}{c}\text { Educational } \\
\text { and } \\
\text { Administrative } \\
\text { Parameters }\end{array}$} & Reporting Tools & $\checkmark$ & $\checkmark$ & $\checkmark$ & $x$ & $\checkmark$ & $\checkmark$ \\
\hline & Rating Tools & $x$ & $\checkmark$ & $\checkmark$ & $x$ & $\checkmark$ & $\checkmark$ \\
\hline & Certificate Manager & $\checkmark$ & $\checkmark$ & $\checkmark$ & $x$ & $\checkmark$ & $\checkmark$ \\
\hline & Virtual Class Manager & $\checkmark$ & $\checkmark$ & $\checkmark$ & $\checkmark$ & $x$ & $\mathrm{x}$ \\
\hline & Cooperation Manager & $\checkmark$ & $\checkmark$ & $\checkmark$ & $\checkmark$ & $\checkmark$ & $\checkmark$ \\
\hline & Lecturers Planner & $\checkmark$ & $\checkmark$ & $\checkmark$ & $\checkmark$ & $x$ & $x$ \\
\hline & User Access Controllers & $\checkmark$ & $\checkmark$ & $\checkmark$ & $\checkmark$ & $\checkmark$ & $\checkmark$ \\
\hline & Course System & $\checkmark$ & $\checkmark$ & $\checkmark$ & $\checkmark$ & $\checkmark$ & $\checkmark$ \\
\hline & Simulations & $x$ & $x$ & $x$ & $x$ & $\checkmark$ & $\checkmark$ \\
\hline & Curriculum Management & $\checkmark$ & $\checkmark$ & $\checkmark$ & $\checkmark$ & $\checkmark$ & $\checkmark$ \\
\hline & Lesson Ratings & $\checkmark$ & $\checkmark$ & $\checkmark$ & $\checkmark$ & $\mathrm{x}$ & $\mathrm{x}$ \\
\hline & $\begin{array}{l}\text { Availability of the } \\
\text { Glossary of Terms }\end{array}$ & $\mathrm{x}$ & $\mathrm{x}$ & $\mathrm{x}$ & $\checkmark$ & $\mathrm{x}$ & $x$ \\
\hline & Development Monitoring & $x$ & $\checkmark$ & $\checkmark$ & $x$ & $x$ & $x$ \\
\hline & Activity Manager & $\checkmark$ & $\checkmark$ & $\checkmark$ & $\checkmark$ & $x$ & $x$ \\
\hline & Quiz Manager & $\checkmark$ & $\checkmark$ & $\checkmark$ & $\checkmark$ & $\checkmark$ & $\checkmark$ \\
\hline & $\begin{array}{l}\text { Education criterion And } \\
\text { Outputs }\end{array}$ & $\mathrm{x}$ & $\checkmark$ & $x$ & $\checkmark$ & $\mathrm{x}$ & $\mathrm{x}$ \\
\hline & Wishlists & $x$ & $\checkmark$ & $x$ & $x$ & $x$ & $x$ \\
\hline & Support Of Webinar & $\mathrm{x}$ & $\mathrm{x}$ & $\mathrm{x}$ & $\mathrm{x}$ & $x$ & $\mathrm{x}$ \\
\hline
\end{tabular}

Table 3: LMS and E - Learning Author Tools Educational and Administrative Parameters Comparison

For 4 LMS platforms have virtual class manager, cooperation manager, lecturer's planner, user Access controllers, course system, simulations, curriculum management, lessons ratings, activity manager, quiz manager, parameters. Adobe Captivate and Articulate Storyline have got all parameters. In LMS and E - Learning Author Tools comparisons, collaboration management, user access, course system, curriculum management, quiz and test management and webinar support are common features.

\begin{tabular}{|c|c|c|c|c|c|c|c|}
\hline & & \multicolumn{4}{|c|}{ Learning Management Systems } & \multicolumn{2}{|c|}{$\begin{array}{l}\text { E - Learning } \\
\text { Author Tools }\end{array}$} \\
\hline & & $\begin{array}{l}\frac{0}{\bar{\delta}} \\
\frac{0}{\Sigma}\end{array}$ & 足 & $\begin{array}{l}\text { 임 } \\
\frac{0}{0} \\
\frac{0}{0} \\
\frac{0}{0} \\
\frac{\pi}{0}\end{array}$ & 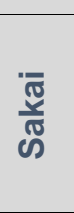 & 造 & 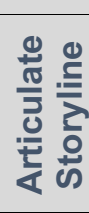 \\
\hline \multirow{5}{*}{$\begin{array}{l}\text { Compatibility } \\
\text { Parameters }\end{array}$} & $\begin{array}{l}\text { Third Party Authoring } \\
\text { Tools }\end{array}$ & $\checkmark$ & $\checkmark$ & $\checkmark$ & $\checkmark$ & $x$ & $x$ \\
\hline & $\begin{array}{l}\text { Third Party } \\
\text { Teleconference Tools }\end{array}$ & $\checkmark$ & $x$ & $x$ & $\checkmark$ & $x$ & $x$ \\
\hline & Tin Can API & $\checkmark$ & $\mathrm{x}$ & $\checkmark$ & $\mathrm{x}$ & $\checkmark$ & $\checkmark$ \\
\hline & $\mathrm{AICC}$ & $\checkmark$ & $x$ & $\checkmark$ & $\mathrm{x}$ & $\checkmark$ & $\checkmark$ \\
\hline & SCORM & $\checkmark$ & $\checkmark$ & $\checkmark$ & $\checkmark$ & $\checkmark$ & $\checkmark$ \\
\hline
\end{tabular}


Tablo 4: LMS and E - Learning Author Tools Compatibility Parameters Comparison

Within the scope of the research, third party author tools and SCORM parameters are similar in 4 LMS platforms. Adobe Captivate and Articulate Storyline comparisons, all parameters are similar. Only SCORM parameters similar for LMS and Author Tools comparisons.

\begin{tabular}{|c|c|c|c|c|c|c|c|}
\hline & & \multicolumn{4}{|c|}{ Learning Management Systems } & \multicolumn{2}{|c|}{$\begin{array}{l}\text { E - Learning } \\
\text { Author Tools }\end{array}$} \\
\hline & & $\begin{array}{l}\frac{0}{\overline{0}} \\
\frac{0}{\Sigma} \\
\Sigma\end{array}$ & 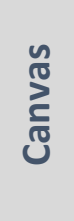 & $\begin{array}{l}\text { 임 } \\
\frac{0}{0} \\
\frac{0}{0} \\
\frac{\pi}{0}\end{array}$ & 离 & 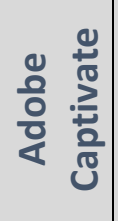 & 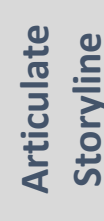 \\
\hline \multirow{3}{*}{$\begin{array}{c}\text { Customizable } \\
\text { Parameters }\end{array}$} & Customizable Fields & $\checkmark$ & $\checkmark$ & $\checkmark$ & $x$ & $\checkmark$ & $\checkmark$ \\
\hline & $\begin{array}{l}\text { Customizable } \\
\text { Functions }\end{array}$ & $x$ & $x$ & $x$ & $\checkmark$ & $\checkmark$ & $\checkmark$ \\
\hline & $\begin{array}{l}\text { Customizable } \\
\text { Reportings }\end{array}$ & $x$ & $\checkmark$ & $x$ & $x$ & $x$ & $x$ \\
\hline
\end{tabular}

Tablo 5: LMS ve E - Learning Author Tools Customizable Parameters Comparison

There are no similarities in the comparison of the 4 LMS platforms, Adobe Captivate and Articulate Storyline, and LMS and E - Learning Author Tools comparisons.

\begin{tabular}{|c|c|c|c|c|c|c|c|}
\hline & & \multicolumn{4}{|c|}{ Learning Management Systems } & \multicolumn{2}{|c|}{$\begin{array}{l}\text { E - Learning } \\
\text { Author Tools }\end{array}$} \\
\hline & & $\begin{array}{l}\frac{0}{\overline{0}} \\
\frac{0}{\Sigma}\end{array}$ & 先 & $\begin{array}{l}\text { 임 } \\
\frac{0}{0} \\
\frac{0}{0} \\
\frac{\pi}{0}\end{array}$ & 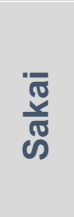 & 选 & 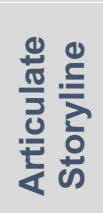 \\
\hline \multirow{6}{*}{$\begin{array}{c}\text { Student } \\
\text { Parameters }\end{array}$} & $\begin{array}{l}\text { Lesson Register } \\
\text { System }\end{array}$ & $\checkmark$ & $\checkmark$ & $\checkmark$ & $\checkmark$ & $\mathrm{x}$ & $\mathrm{x}$ \\
\hline & $\begin{array}{l}\text { Self Controlled } \\
\text { Education }\end{array}$ & $\checkmark$ & $\checkmark$ & $\checkmark$ & $\mathrm{x}$ & $\mathrm{x}$ & $\mathrm{x}$ \\
\hline & Social Learning & $\checkmark$ & $\checkmark$ & $\checkmark$ & $\checkmark$ & $x$ & $x$ \\
\hline & Student Manager & $\checkmark$ & $\checkmark$ & $\checkmark$ & $\checkmark$ & $x$ & $x$ \\
\hline & $\begin{array}{l}\text { Student Register } \\
\text { System }\end{array}$ & $\checkmark$ & $\checkmark$ & $\checkmark$ & $\checkmark$ & $\mathrm{x}$ & $\mathrm{x}$ \\
\hline & Student Portal & $\checkmark$ & $\checkmark$ & $\checkmark$ & $\checkmark$ & $x$ & $\mathrm{x}$ \\
\hline
\end{tabular}

Table 6: LMS and E - Learning Author Tools Student Parameters Comparison

In the comparison of student parameters, only self-controlled education differs in Sakai. Adobe Captivate and Articulate Storyline comparisons show that all parameters are similar. The differences between LMS and E - Learning Author Tools are only significant. 


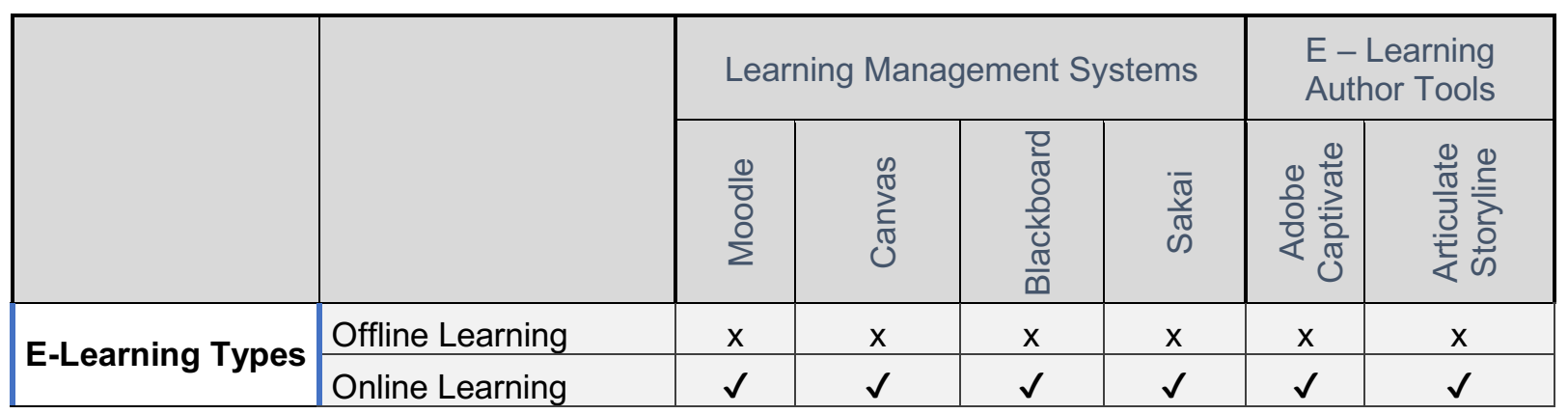

Tablo 7: LMS and E - Learning Author Tools E-Learning Types Comparison

All of comparisons have same similarities for about LMS and author tools. With this context; there are differecies for only main parameters of E-Learning and custmizable.

\begin{tabular}{|c|c|c|c|c|c|c|c|}
\hline & & \multicolumn{4}{|c|}{ Learning Management Systems } & \multicolumn{2}{|c|}{$\begin{array}{l}\text { E - Learning } \\
\text { Author Tools }\end{array}$} \\
\hline & & $\begin{array}{l}\frac{0}{\overline{0}} \\
\frac{0}{\Sigma}\end{array}$ & 疍 & $\begin{array}{l}\text { 힘 } \\
\frac{0}{0} \\
\frac{0}{0} \\
\frac{0}{0} \\
\frac{\pi}{n}\end{array}$ & 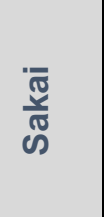 & 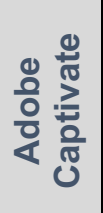 & 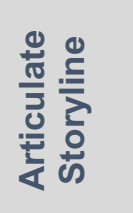 \\
\hline \multirow{3}{*}{ Learning Model } & $\begin{array}{l}\text { Synchronous } \\
\text { Learning }\end{array}$ & $\mathrm{x}$ & $\mathrm{x}$ & $\checkmark$ & $\checkmark$ & $\mathrm{x}$ & $\mathrm{x}$ \\
\hline & $\begin{array}{l}\text { Asynchronous } \\
\text { Learning }\end{array}$ & $\checkmark$ & $\checkmark$ & $\checkmark$ & $\checkmark$ & $\checkmark$ & $\checkmark$ \\
\hline & Blended Learning & 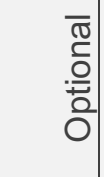 & $\begin{array}{l}\overline{\bar{\pi}} \\
\overline{0} \\
. \overline{0} \\
0 \\
0\end{array}$ & $\begin{array}{l}\bar{\pi} \\
\overline{0} \\
\overline{0} \\
\overline{0}\end{array}$ & 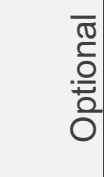 & 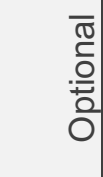 & 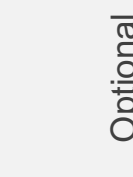 \\
\hline
\end{tabular}

\section{Tablo 8: LMS ve E - Learning Author Tools Learning Model Comparison}

In the comparison of learning model parameters, asynchronous learning and blended learning are similar in both 4 LMS platforms and Adobe Captivate and Articulate Storyline comparisons.

\section{Conclusion}

In this study, the extent to which the platforms and tools that online education programs are offered in the context of the problems experienced in the online education programs which are preferred by the elite educational institutions nowadays cover the developing technologies and what kind of solutions can be produced for these platforms and tools have been surveyed. Comparative content analysis was used as a method in this research identified 8 main parameters and 66 sub parameters with online education platforms and tools literature survey. With the identified parameters, reached the following findings have been reached.

The content analysis of Moodle, Canvas, Blacboard and Sakai LMS platforms showed similarity with $58 \%$. Adobe Captivate and Articulate similarities of Storyline E - Learning Author Tools are examined, it is seen that this rate is much higher as $95 \%$. Similarity rate was $79 \%$ for Moodle Canvas comparison, $83 \%$ for Moodle - Blacboard comparison, $74 \%$ for Moodle - Sakai comparison, $76 \%$ for Canvas - Blacboard comparison, $71 \%$ for Cnavas - Sakai comparison and finally for Blackboard - Sakai. It is seen that the similarity rate is $68 \%$. In this context, the first hypothesis "Platforms of using online education programs have similar features and capabilities" and the sixth hypothesis "Authoring tools software's for produce online education programs have similar features and capabilities" confirmed. 
There is no evidence that LMS platforms support innovative media approaches such as virtual reality, augmented reality, blended reality. Therefore, second hypothesis "Platforms of using online education programs have innovative media technologies like virtual reality, augmented reality, mixed reality" and fifth hypothesis "Authoring tools software's for produce online education programs have not innovative media technologies like virtual reality, augmented reality, mixed reality" confirmed.

Support of webinar parameters is not containing LMS or Author Tools. This situation confirming third hypothesis of "Platforms of using online education programs have limitations of live streaming like webinar".

In the LMS platforms and E - Learning Author Tools, mobile application is only available on Moodle and Blackboard platforms. With this finding fourth hypothesis "Platforms of using online education programs have limitations of mobile applications" partially confirmed.

As regards the e-learning standards, only the content produced in Tin Can API, AICC and Scorm standards were found to be compatible. With this finding seventh hypothesis "Authoring tools software's for produce online education programs have not compatibility issues" partially unconfirmed. In this context eighth hypothesis of research "Authoring tools software's for produce online education programs and platforms of using online education programs; compatible with online education standards like SCORM and AICC" confirmed.

\section{References}

[1] Arslan, M. (2009). Eğitim Bilimine Giriş, Ankara: Gündüz Education and Publishing.

[2] Aslan, T. (2013). Uzaktan Eğitim ve Öğrenme Yönetim Sistemlerinin Karşılaştırılması. Yüksek Lisans Tezi, Trakya Üniversitesi institute of science and technology, Fizik Anabilim Dalı, Edirne.

[3] Aşkar, P. (2003). Uzaktan Eğitimde Temel Yaklaşımlar ve Uzaktan Eğitimde Öğrenci (Katılımcı) Olmak. Editör: Ali Tahran. Uzaktan Eğitim Teknolojileri ve TCMB'de Teknoloji Destekli Bilgisayar Eğitimi Konferansı, Ankara: TCMB, p: 3-40.

[4] Bayram, F. İbili, E. Hakkari, F. Kantar, M. Doğan, M. (2009). E-Üniversite: SCORM Uyumlu Modüler Öğrenim Yönetim Sistemlerinin Yükseköğretimde Kullanımı. XI. Akademik Bilişim Konferansı. Harran University, Şanlıurfa.

[5] Beynon, M. (2007). Computing technology for learning - in need of a radical new conception. Educational Technology \& Society, Issue: 10, Number: 1, 94-106.

[6] Campanella, S. Dimauro, G. Ferrante, A. Impedovo, D. Impedovo, S. Lucchese, M. G. Modugno, R. Pirlo, G. Sarcinella, L. Stasolla, E. Trullo, C. A. (2008). E-learning platforms in the Italian Universities: the technological solutions at the University of Bari. Wseas Transactions on Advances in Engineering Education, Issue: 1, Volume: 5.

[7] Clark, R. E. (2001). A Summary of Disagreements With The 'Mere Vehicles' Argument. Learning From Media: Arguments, Analysis, and Evidence. Greenwich, CT: Information Age Publishing, (2nd Edition).

[8] Engin, M. (2013). Üniversitelerde Teknoloji Yoğun Uzaktan Eğitim Sistemlerinin Üretim, Uygulama ve Yönetim Süreçlerinin İncelenmesi. Doktora Tezi. Ankara Üniversitesi, Eğitim Bilimleri Enstitüsü Bilgisayar ve Öğretim Teknolojileri Eğitimi Anabilim Dalı, Eğitim Teknolojisi Programı. Ankara.

[9] Epignosis LLC (2014). E-Learning Concepts, Trends, Applications, California: Epignosis LLC

[10] İşman, A. (2011). Uzaktan Eğitim, Ankara: Pagem Akademi Publishing.

[11] Moore, M. G. Kearsley, G. (1996). Distance Education: A Systems View. Boston, MA: Wadsworth Publishing.

[12] Özkeskin, E. E. (2007). Kalıcılığa Olumlu Etkisi Tanımlanmış Bir Bilgisayar Destekli Öğretim Materyalinin SCORM Uyumlu Hale Getirilmesi. Yüksek Lisans Tezi, Çukurova Üniversitesi Sosyal Bilimler Enstitüsü, Bilgisayar ve Öğretim Teknolojileri Eğitimi Anabilim Dalı, Adana.

[13] Preece, J., Maloney-Krichmar, D. and Abras, C. (2003) History of Online Communities, In Karen Christensen \& David Levinson (Eds.), Encyclopedia of Community: From Village to Virtual World. Thousand Oaks: Sage Publications. 
[14] Saba, F. (2003). Distance Education Theory, Methodology, and Epistemology: A Pragmatic Paradigm, Handbook of Distance Education, Editors: Moore, M. G. Anderson, W. G. London: Lawrence Erlbaum Associates Publishers.

[15] Schlosser, C. A. Anderson, M. L. (1994). Distance Education: Review of the Literature. Lowa: IDEA Publishing.

[16] Şimşek, N. (2012). Uzaktan Eğitimde Kalite Göstergeleri ve Teknoloji Temelli Uzaktan Eğitimin Bu Göstergeler Açısından Değerlendirilmesi. Eğitim Bilimleri ve Uygulama Dergisi, Cilt: 11, Sayı: 21, SS: $1-24$.

[17] Şişman, M. (2007). Eğitim Bilimine Giriş, Ankara: Pagem Akademi Yayınları.

[18] Watson, W.R. Watson, S.L. (2007). An Argument for Clarity: What are Learning Management Systems, What are They Not, and What Should They Become? TechTrends, Issue: 51, Number: 2

[19] Edutechnica: http://edutechnica.com/ (Access Date: 05.03.2019).

[20] E-Learning Industry: https://elearningindustry.com/learning-management-systemscomparison-checklist-of-features (Access Date: 01.03.2019).

[21] Growth Engineering: https://www.growthengineering.co.uk/what-is-aicc/ (Access Date: 01.03.2019).

[22] Trivantis: https://www.trivantis.com/ (Access Date: 05.03.2019).

[23] Uzaktan Eğitim, Öğrenme Yönetim Sistemi (LMS): https://ueytulms.wordpress.com/2016/12/21/scorm-nedir-ozellikleri-ve-bilesenleri-nelerdir/ (Access Date: 05.03.2019).

[24] Market Insight Reports: https://www.marketinsightsreports.com/reports/05281261168/globalelearning-authoring-tools-market-size-status-and-forecast-2019-

2025? source=Industryreports24\&Mode=3 (Access Date: 05.03.2019). 